\title{
Ophthalmologic Manifestations as Initial Presentation of Patients with Chronic Myeloid Leukemia: Report of Two Cases
}

\author{
Shehab F. Mohamed ${ }^{a} \quad$ Ahmed Qatami $^{b} \quad$ Abdulqadir Nashwan $^{a}$ \\ Mohammad A. Abdulla ${ }^{a}$ Mohamed A. Yassin ${ }^{a}$ \\ aHematology, Medical Oncology Section, National Center for Cancer Care and Research, \\ Hamad Medical Corporation (HMC), Doha, Qatar; ${ }^{b}$ Ophthalmology Department, Hamad \\ Medical Corporation (HMC), Doha, Qatar
}

\section{Keywords}

Chronic myeloid leukemia · Ophthalmologic manifestations · Cancer biology · Drug therapy ·

\begin{abstract}
Chronic myeloid leukemia (CML) is a myeloproliferative neoplasm, classically described as triphasic disease (chronic, accelerated, and blast). Despite the significant efforts made by different groups to optimize treatment and outcome, there are still unmet needs and unanswered questions. Ophthalmologic manifestations are among the therapeutic challenges. The best available therapy for patients presenting with ophthalmologic manifestations is still unclear. Here we present two cases of CML (chronic phase) with ophthalmologic manifestations as initial presentation, trying to shed light on this important type of presentation and proposing recommendations for hematologists in view of the current literature.
\end{abstract}

(C) 2020 The Author(s)

Published by S. Karger AG, Basel

\section{Introduction}

In 2016, the WHO revised the classifications for hematopoietic and lymphoid tissue. With regards to myeloproliferative neoplasm, minimal changes were made (prefibrotic myelofibrosis was added as distinct entity and mastocytosis was removed from the list of myeloproliferative neoplasm), the chronic leukemias (chronic neutrophilic leukemia CSF3R mutation identified as oncogene and for chronic myeloid leukemia [CML] and chronic eosinophilic leukemia) remain unchanged in comparison to previous classifications (WHO 2008).

Prof. Shehab F. Mohamed 
Mohamed et al.: Ophthalmologic Manifestations as Initial Presentation of Patients with Chronic Myeloid Leukemia
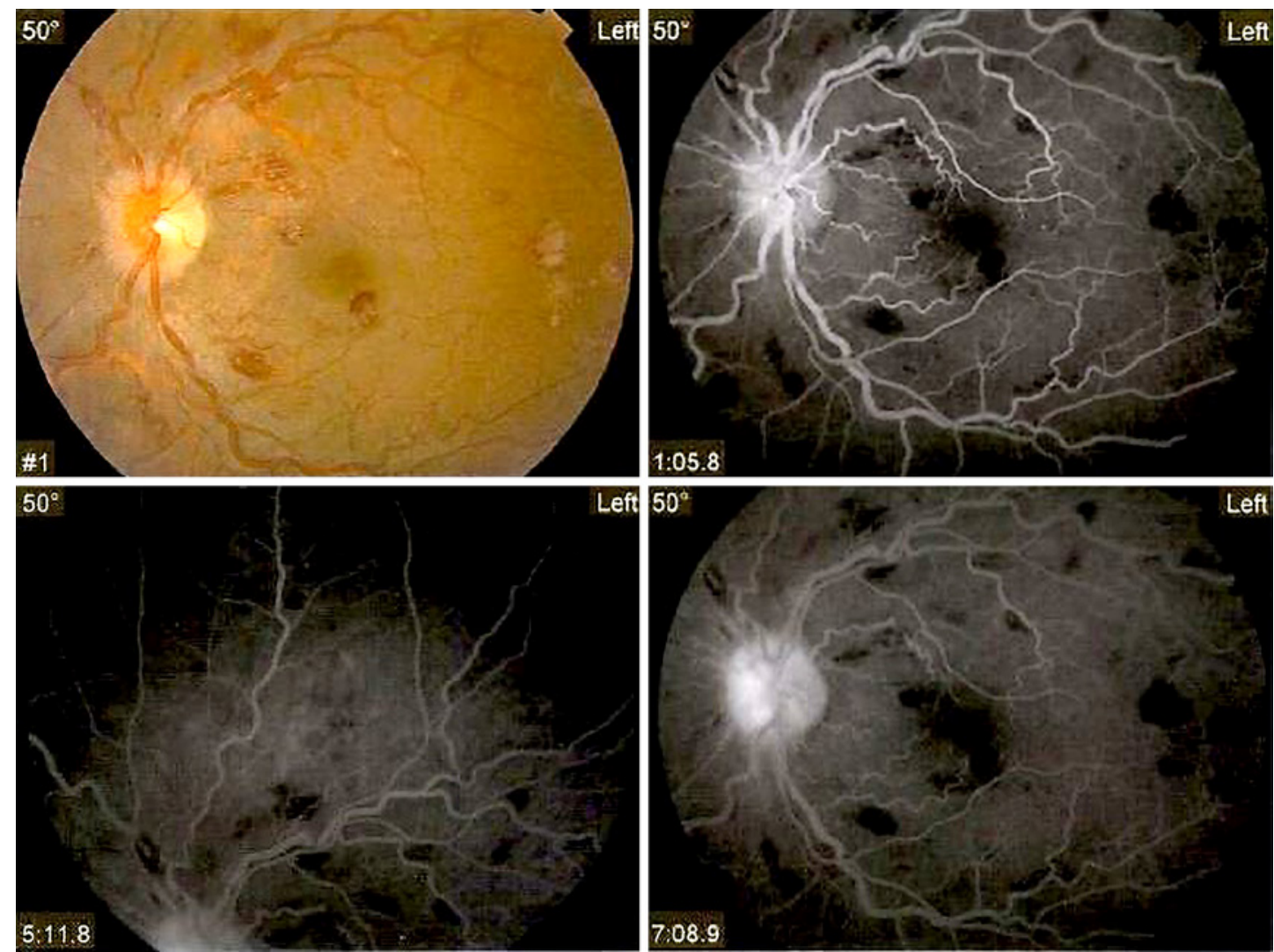

Fig. 1. Fundus photograph showing scattered intraretinal hemorrhages.

CML is a myeloproliferative neoplasm that can present in different ways; it varies from incidental finding in routine complete blood count to symptomatic presentations such as splenomegaly.

Among the uncommon presentations of CML are ophthalmologic manifestations, which can vary from incidental finding during eye examination to blurred vision and partial/total loss of vision.

\section{Case Presentation}

\section{Case 1}

A 31-year-old Egyptian male, who was previously healthy apart from refractive error and wearing glasses, presented with a condition that started with a 1-month history of blurred vision in his right eye. He was referred to ophthalmology, and fundoscopy showed bilateral retinal hemorrhages (Fig. 1).

Hematology routine investigation showed a high white blood cell (WBC) count of more than $400 \times 10^{3} / \mu \mathrm{L}$ (normal range: $4-10 \times 10^{3} / \mu \mathrm{L}$ ); he underwent peripheral smear, bone marrow was suggestive of CML, cytogenetics were abnormal (Philadelphia chromosome), and BCR ABL by PCR was positive (84\%), which confirmed the diagnosis of CML (chronic phase). The patient received nilotinib (300 $\mathrm{mg}$, oral, b.i.d.) as an upfront therapy after cytoreduction with hydroxyurea. His ophthalmologic follow-up after 2 years of treatment showed significant improvement (Fig. 2). 
Mohamed et al:: Ophthalmologic Manifestations as Initial Presentation of Patients with Chronic Myeloid Leukemia
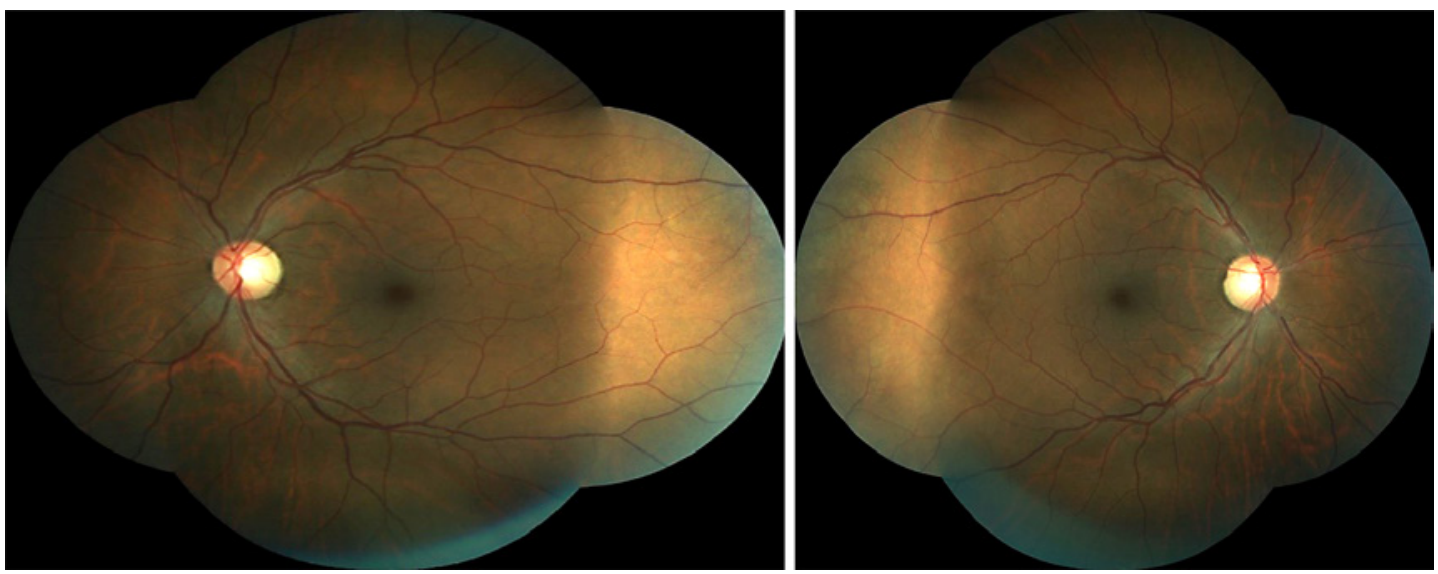

Fig. 2. Normal fundus (bilaterally) after treatment.

Case 2

A 35-year-old male completed his treatment for tuberculosis 1 month prior to his admission. He developed sudden blurred vision followed by a transient loss of vision with no conjunctival bleeding. His ophthalmologic check revealed left-eye resolved disc edema and residual foveal mottling.

His complete blood count was $276.7 \times 10^{3} / \mu \mathrm{L}$ (normal range: $4-10 \times 10^{3} / \mu \mathrm{L}$ ); peripheral smear and bone marrow were suggestive of CML, cytogenetics were abnormal (Philadelphia chromosome), and BCR ABL by PCR was positive (90\%), which confirmed the diagnosis of CML. The patient started initially on hydroxyurea as cytoreduction followed by nilotinib (300 mg, oral, b.i.d.) as an upfront therapy.

\section{Discussion}

Ophthalmic manifestations are uncommon presentations of CML with a paucity of literature, and little is known about it. How does it present? Do we need to do ophthalmic examination for each patient diagnosed with CML? Is there a cutoff for leukocytosis which can predict eye damage? Both hematologists and ophthalmologists should work together to achieve a better outcome and to decide about the best available therapy.

Ophthalmologic manifestations have a wide range of presentations and findings upon fundoscopy and eye examination. The optic nerve or retina might be affected. The pathological complications on the eye could be reversible or irreversible. The irreversible could be urgent like retinal hemorrhage or non-urgent like cotton wool spots, infections, or proptosis.

Blurring of vision or blindness could be the first presentation of CML; in other scenarios, it could also be the first presentation of blast crisis. Patients with ophthalmic manifestation of CML have been reported to have a lower 5-year survival rate [1].

An increased level of WBC, such as 300,000, will present with a picture of hyperviscosity. Fundoscopy of these patients might reveal papilledema, venous obstruction, and hemorrhages [2].

Features of leukemic retinopathy include yellowish-white masses of variable size, multiple pre-retinal and intraretinal hemorrhages, cotton wool spots, perivascular sheathing, and neovascularization. Roth's spot hemorrhages may represent small areas of retinal leukemic infiltration. Leukemic retinopathy is more common in the acute leukemias and is usually a poor prognostic indicator. 
Mohamed et al:: Ophthalmologic Manifestations as Initial Presentation of Patients with Chronic Myeloid Leukemia

Peripheral retinal changes, such as neovascularization reminiscent of "sea fans" or microaneurysm resulting from peripheral non-perfusion, tend to occur in patients with chronic disease.

Leukemic infiltrates most often affect the choroid; however, these are difficult to detect clinically and may present with a serious or exudative retinal detachment. Additionally, pallor and swelling of the optic nerve may indicate optic nerve infiltration and require immediate referral for imaging studies.

As we can see our two cases presented with blurring of vision, and one of them was found to have hemorrhage and the other disc edema.

Leukocytosis can lead to hyperviscosity and ophthalmic manifestations; however, there is no cutoff number (leukocyte count) that can predict this. There is no consensus on how to treat these patients, but most of them are treated with both surgery and medication (cytoreduction with or without TKI). Leukemic infiltration of the eye is best treated with systemic chemotherapy appropriate for the type and stage of the leukemia. Adjunct radiation may be applied to lesions of the optic nerve or orbit. Of note, the various chemotherapeutic agents used to treat leukemia may cause ocular toxicity such as cataract development, cranial nerve palsies, optic atrophy, and intraocular inflammation [3-13].

We believe that early intervention by medication cannot only save the patient's eye but might also prevent the patient from undergoing surgery, as we can see in both our patients who improved with medications only.

\section{Conclusion}

Ophthalmic manifestation can be the first presentation of CML which can lead to irreversible damage; it is associated with a high WBC level on presentation.

We recommend baseline fundoscopy and eye examination by an ophthalmologist for all newly diagnosed CML.

\section{Acknowledgment}

The authors acknowledge support from the Qatar National Library.

\section{Statement of Ethics}

Ethical approval has been obtained from Hamada Medical Research Center, and informed consent was obtained according to our institutes' committee (ABHATH). Our research complies with the guidelines for human studies and was conducted ethically in accordance with the World Medical Association Declaration of Helsinki.

\section{Disclosure Statement}

The authors have no conflict of interest to declare.

\section{Funding Sources}

The publication of this article was funded by Qatar National Library. 
Mohamed et al.: Ophthalmologic Manifestations as Initial Presentation of Patients with Chronic Myeloid Leukemia

\title{
Author Contributions
}

\author{
All authors contributed equally.
}

\section{References}

1 Alegre A, Ojeda E, Mataix R, Molero T. Ocular infiltration as a presenting form of extramedullary blast crisis in CML. Sangre. 1990;35(3):236-7.

2 Lipton JH, Mcgowan HD, Payne DG. Ocular masquerade syndrome in lymphoid blast crisis of chronic myeloid leukemia. Leuk Lymphoma. 1995;20(1-2):161-3.

3 Huynh TH, Johnson MW, Hackel RE. Bilateral proliferative retinopathy in chronic myelogenous leukemia. Retina. 2007;27(1):124-5.

4 Mohamed SF, Qatami A, Nashwan A, Yassin MA. Ophthalmic manifestations of chronic myeloid leukemia as initial presentation and unmet needs. Blood. 2018 Nov;132(Suppl 1):5454.

5 Mandava N, Costakos D, Bartlett HM. Chronic myelogenous leukemia manifested as bilateral proliferative retinopathy. Arch Ophthalmol. 2005;123:576-7.

6 Nobacht S, Vandoninck KF, Deutman AF, Klevering BJ. Peripheral retinal nonperfusion associated with chronic myeloid leukemia. Am J Ophthalmol. 2003;135:404-6.

7 Jackson N, Reddy SC, Hishamuddin M, Low HC. Retinal findings in adult leukaemia: correlation with leukocytosis. Clin Lab Haematol. 1996;18:105-9.

8 Hasanreisoğlu B, Or M, Atmaca LS, Haznedar R. Pars plana vitrectomy in chronic myelogenous leukemia with vitreous hemorrhage. Jpn J Ophthalmol. 1988;32(3):304-9.

9 Costagliola C, Rinaldi M, Cotticelli L, Sbordone S, Nastri G. Isolated optic nerve involvement in chronic myeloid leukemia. Leuk Res. 1992;16(4):411-3.

10 Mandić BD, Potocnjak V, Bencić G, Mandić Z, Pentz A, Hajnzić TF. Visual loss as initial presentation of chronic myelogenous leukemia. Coll Antropol. 2005;29(Suppl 1):141-3.

11 Huang PK, Sanjay S. Visual disturbance as the first symptom of chronic myeloid leukemia. Middle East Afr J Ophthalmol. 2011 Oct;18(4):336-8.

12 Kapadia RK, Steeves JH. Roth spots in chronic myelogenous leukemia. CMAJ. 2011 Dec;183(18):E1352.

13 Macedo MS, Figueiredo AR, Ferreira NN, Barbosa IM, Furtado MJ, Correia NF, et al. Bilateral proliferative retinopathy as the initial presentation of chronic myeloid leukemia. Middle East Afr J Ophthalmol. 2013 Oct-Dec; 20(4):353-6. 